\title{
ARCHIV DER PHARMACIE。
}

CC. Bandes erstes Heft.

\section{A. Originalmittheilungen. \\ I. Chemie und Pharmacie.}

\section{Ueber den Euxenit von Hitteröe.}

Von Dr. Carl Jehn in Geseke.

$\mathrm{Zu}$ den Mineralien, welche Yttrium, Cer und die damit verwandten Metalle als Oxyde und $\mathrm{Niob}$ mit seinen Begleitern als Säuren enthalten, gehört auch der Euxenit. Anfangs 1871 erhielt ich einige Gramme Euxenit von einem neuen Fundorte, von der Insel Hitteröe bei Norwegen, durch Herrn Prof. Dr. G. vom Rath in Bonn, welcher mir denselben zur qualitativ-quantitativen Untersuchung freundlichst überliess. Von den früheren Analysen des Euxenits sind zu nennen die von Strecker, Chydenius und von D. Forbes und Dahle, welche jedoch sämmtlich bedeutend differiren. Ich übergehe dieselben und beschränke mich darauf, eine Analyse aus der letzten Zeit anzuführen.

Dieselbe rührt von Behrend her (Ber. d. deutsch. chem. Gesellschaft 1869), welcher den Euxenit von Eydland bei Lindernäs in Norwegen untersuchte und folgende Zahlen fand:

\begin{tabular}{lr}
$\mathrm{Nb}^{2} \mathrm{O}^{5}$ & $31,98 \%$ \\
$\mathrm{TiO}^{2}$ & $19,17 \%$ \\
$\mathrm{UO}$ & $19,52 "$ \\
$\mathrm{YO}$ & $18,23 "$ \\
$\mathrm{CeO}$ & $2,84 \%$ \\
$\mathrm{FeO}$ & $4,77 \%$ \\
$\mathrm{CaO}$ & $1,19 "$ \\
$\mathrm{Alkalien}$ & $0,82 \%$ \\
$\mathrm{H}^{2} \mathrm{O}$ & $2,40 \%$ \\
\cline { 2 - 2 } & $\mathbf{1 0 0 , 9 2 \%}$
\end{tabular}

Arch. d, Pharm. GC. Bds, 1, Hft. 
Herr Professor Rammelsberg leitet ans obiger Analyse für den Euxenit folgende Formel ab:

$$
\begin{aligned}
& \mathrm{R}^{4} \mathrm{Nb}^{2} \mathrm{Ti}^{2} \mathrm{O}^{13}+\mathrm{H}^{2} \mathrm{O} \text { oder }
\end{aligned}
$$

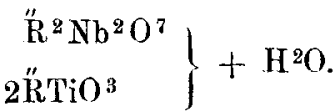

Der mir zur Untersuchung übergebene Euxenit von Hitteröe war schwarz, in dïnnen Schichten rothbraun durchscheinend, zeigte einen muschligen Bruch und liess sich ziemlich leicht pulvern. Eine qualitative Analyse stellte die Anwesenheit nachstehender Stoffe fest:

$$
\mathrm{Nb}^{2} \mathrm{O}^{5}, \mathrm{TiO}^{2}, \mathrm{UO}, \mathrm{CeO}, \mathrm{YO}, \mathrm{CaO}, \mathrm{Al}^{2} \mathrm{O}^{3}, \mathrm{FeO}, \mathrm{MgO}
$$

und Spuren von $\mathrm{K}^{2} \mathrm{O}$ und $\mathrm{Na}^{2} \mathrm{O}$.

Zur Bestimmung des Wassergehaltes wurde die fein zerriebene und gebentelte Substanz im Exsiccator über $\mathrm{CaCl}^{2}$ getrocknet und dann 4 Stunden lang im Trockenapparate bei $100^{\circ} \mathrm{C}$. gehalten; es trat keine Gewichtsverminderung ein. Hierauf wurde sie in ein Porzellanschiffchen gegeben und in einer Glasröhre 2 Stunden lang im $\mathrm{CO}^{2}$ Strome geglüht. Als Mittelwerth von zwei auf diese Weise ausgeführten Bestimmungen ergab sich der Wassergehalt des Euxenits zu 2,87\%. Hierauf ging ich zur vollständigen quantitativen Analyse ïber.

Das fein gepulverte Mineral wurde in einer zugeschmolzenen Glasröhre mit einem Gemisch von 4 Vol. conc. $\mathrm{H}^{2} \mathrm{SO}^{4}$ und 3 Vol. $\mathrm{H}^{2} \mathrm{O} 12$ Stunden lang im Luftbade auf circa $250^{\circ} \mathrm{C}$. erhitzt. Nach Verlauf dieser Zeit war die $\mathrm{Nb}^{2} \mathrm{O}^{5}$ als ein rein weisses Pulver am Boden der Röhre abgeschieden. Letztere wurde geöffnet, der Inhalt derselben in eine geräumige Schale mit Wasser gespült, die Schwefelsäure mit Ammoniak fast neutralisirt und nunmehr durch anhaltendes Kochen die $\mathrm{TiO}^{2}$ gefällt. Die Trennung der $\mathrm{Niob}$ - und Titaṇsäure wurde mit einer verdünnten KHO-Lösung bewirkt, wobei sich erstere löste. Aus der stark verdünnten alkalischen Lösung wurde kochend die $\mathrm{Nb}^{2} \mathrm{O}^{5}$ mit $\mathrm{H}^{2} \mathrm{SO}^{4}$ gefällt. Der Niederschlag ist eine Verbindung von Niobsäure und Schwefelsäure. Zur Beseitigung der letzteren warde er unter Zusatz von Ammoniumcarbonat stark geglüht. - 
Die von $\mathrm{Nb}^{2} \mathrm{O}^{5}$ und $\mathrm{TiO}^{2}$ befreite, schwachsaure Flüssigkeit wurde mit $\mathrm{C}^{2} \mathrm{H}^{3} \mathrm{NaO}^{2}$ versetzt, so dass sie nur noch freie Essigsäure enthielt, und dann mit Ammoniumoxalat. Die gefällten Oxalate von Kalk, Yttriumoxyd and Ceroxydul wurden abfiltrirt, mit verdünnter Ammoniumoxalatlösung ausgewaschen, getrocknet und zur Zerstörung der $\mathrm{C}^{2} \mathrm{H}^{2} \mathrm{O}^{4}$ geglüht. Der aus Kalk, Yttriumoxyd und Ceroxyd bestehende Rückstand wurde in $\mathrm{HCl}$ gelöst, und aus der verdünnten Lösung durch kohlensäurefreies Ammoniak Ceroxyd und Yttererde gefällt. Der Niederschlag wurde mit etwas $\mathrm{H}^{2} \mathrm{SO}^{4}$ aufgenommen, die Lösung mit KHO fast neutralisirt und dann mit einer gesättigten Tösung von neutralem Kaliumsulfat versetzt. Nach 24 Stunden wurde das ausgeschiedene Cerdoppelsalz abfiltrirt, mit Kaliumsulfatlösung ausgewaschen, in kochend heissem, schwach $\mathrm{HCl}$-haltigen Wasser gelöst und wieder mit $\mathrm{C}^{2} \mathrm{H}^{2} \mathrm{O}^{4}$ als Ceroxalat gefällt. Wegen der Anwesenheit der ziemlich bedeutenden Menge von Kali wurde dor Niederschlag abermals in $\mathrm{HCl}$ gelöst und mit Ammoniumoxalat gefällt. (Vorgeschlagen von Rose-Finkener, S. 69.)

Das abfiltrirte und gut ausgewaschene Ceroxalat wurde im bedeckten Tiegel über dem Gasgebläse geglüht. Der Glührückstand besteht, wie zuerst Bunsen nachgewiesen hat, aus $\mathrm{Ce}^{3} \mathrm{O}^{4}$.

Das-Filtrat, welches das Yttriumkaliumdoppelsulfat enthielt, wurde mit Ammoniumoxalat versetzt und 24 Stunden lang sich selbst überlassen. Der Niederschlag, ein Doppelsalz von Kaliumoxalat und Yttriumoxalat, wurde abfiltrirt und durch Glïhen in Kaliumcarbonat und Yttriumoxyd verwandelt. Eine Trennung beider durch Auswaschen mit Wasser ist nicht leicht $\mathrm{zu}$ bewerkstelligen, da das geglühte Yttriumoxyd sich in einem so fein zertheilten Zustande befindet, dass es sich nur sehr schwierig abfiltriren lässt. Ich löste desshalb nach dem Vorschlage von Th. Scheerer (RoseFinkener, S. 64.) das Gemenge in Salpetersäure und fällte die Yttererde mit Ammoniak. Der gut ausgewaschene und getrocknete Niederschlag wurde stark geglüht und als YO 
bestimmt. Die obenerwähnte Lösung der drei Erden enthielt jetzt nur noch $\mathrm{Kalk}$, welcher auf die gewöhnliche Weise als Calciumoxalat gefällt, durch Glühen über dem Gasgebläse in $\mathrm{CaO}$ verwandelt und als solcher gewogen wurde. Die auf angegebene Weise von Säuren und den genannten Erden befreite ursprüngl. Flüssigkeit wurde, um das Uranoxydul in Lösung zu halten, mit überschüssigem Ammoniumcarbonat und dann mit Schwefelammonium versetzt. Die Fällung von Thonerdehydrat und Eisensulfür wurde abfiltrirt, in $\mathrm{HCl}$ gelöst und zur Oxydation des Eisens mit einigen Tropfen HNO ${ }^{3}$ behandelt.

Aus der Lösung wurde mit Kalilange das Eisen gefällt und als $\mathrm{Fe}^{2} \mathrm{O}^{3}$ bestimmt. Das Filtrat wurde mit $\mathrm{HCl}$ schwach angesänert und mit Ammoniak die Thonerde abgeschieden. Die Flüssigkeit, welche jetzt nur noch Uran und Magnesia enthielt, wurde mit $\mathrm{HCl}$ übersättigt, durch Eindampfen concentrirt, vom ausgeschiedenen Schwefel durch Filtration befreit, mit Ammoniak übersättigt, dann mit Ammoniumsulfid versetzt, und eine halbe Stunde lang gekocht. Der hierdurch erzeugte Niederschlag von Uranoxydul kann kleine Mengen von Schwefelammonium enthalien. Derselbe wurde abfiltrirt und mit $\mathrm{H}^{2} \mathrm{O}$ ausgewaschen, dem etwas Schwefelammonium und Chlorammonium hinzugefïgt war.

Nach dem Trocknen wurde das Uranoxydul in einem Rose'schen Tiegel im Wasserstoffstrome stark geglüht und als UO bestimmt. Die Fliissigkeit enthielt jetzt nur noch Magnesia, welche anf die gewöhnliche Weise als Ammoniummagnesiumphosphat gefällt und als Magnesiumpyrophosphat bestimmt wurde.

Als Mittelwerth von drei, auf angegebene Weise ausgeführten Analysen erhielt ich für die Zusammensetzung des Euxenits von Hitteröe folgende 7:ahlen: 


$\begin{array}{lr}\mathrm{Nb}^{2} \mathrm{O}^{5} & \mathbf{1 8 , 3 7 \%} \\ \mathrm{TiO}^{2} & 34,96 " \\ \mathrm{Al}^{2} \mathrm{O}^{3} & 5,41 " \\ \mathrm{EeO} & 2,54 " \\ \mathrm{CaO} & 1,63 " \\ \mathrm{CeO} & 8,43 " \\ \mathrm{YO} & 13,20 " \\ \mathrm{UO} & 7,75 \% \\ \mathrm{MgO} & 3,92, \\ \mathrm{H}^{2} \mathrm{O} & 2,87 \% \\ & 99,08 \% .\end{array}$

Es berechnet sich demnach das Verhältniss der verschiedenen Bestandtheile folgendermassen:

$$
\begin{aligned}
& \mathrm{Nb}^{2} \mathrm{O}^{5}=\frac{1837}{268}=6,48 \\
& \mathrm{TiO}^{2}=\frac{3496}{82}=42,63 \\
& \mathrm{Al}^{2} \mathrm{O}^{3}=\frac{541}{103}=5,25 \\
& \mathrm{FeO}=\frac{254}{72}=3,53 \\
& \mathrm{CaO}=\frac{163}{56}=2,91 \\
& \mathrm{CeO}=\frac{843}{108}=7,81 \\
& \mathrm{YO}=\frac{1320}{803}=16,44 \\
& \mathrm{CO}=\frac{775}{136}=5,70 \\
& \mathrm{MgO}=\frac{392}{40}=9,80
\end{aligned}
$$

Es ist ferner $\mathrm{zu}$ berücksichtigen, dass das $\mathrm{H}^{2} \mathrm{O}$ im Euxenit höchst wahrscheinlich nicht als Krystallwasser, sondern als sogenanntes basisches Wasser vorhanden ist. Es ist somit zu den Basen hinzu zu addiren: 


$$
\mathrm{H}^{2} \mathrm{O}=\frac{.287}{18}=15,94 \text {. }
$$

Wenn wir nun bei der Ueberzahl der zweiwerthigen Elemente alle gefundenen Zahlen auf Zweiwerthigkeiten zurückführen, so müssen wir die vierwerthige $\mathrm{Nb}^{2} \mathrm{O}^{5}$ mit 2 multipliciren. Wir erhalten alsdann $\mathrm{Nb}^{2} \mathrm{O}^{5}=12,96$. Die Summe der für die Säuren gefundenen Zahlen ist, wenn wir dic Thonerde mit zu den Säuren rechnen und als zweiwerthig ansehen $=60,84$, der für die Basen gefundenen Zahlen $=62,13$.

Es herrscht somit, wenn man die Schwierigkeit, die einzelnen Basen scharf zu trennen, in Erwägung zieht, eine genügende Uebereinstimmung. Man sieht, dass wir im Hitteröer Euxenit ein Salz-Gemenge haben, für welches, da das Verhältniss der Aluminate, Niobate und Titanate zu einander ungefähr wie $2: 5: 15$ ist, folgende Formel aufgestellt werden kann:

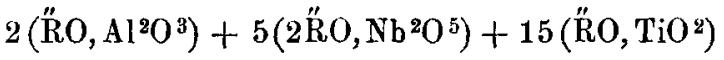

$$
\begin{aligned}
& \text { worin } \ddot{\mathrm{R}}=\mathrm{Fe}, \mathrm{Ca}, \mathrm{Ce}, \mathrm{Y}, \mathrm{U}, \mathrm{Mg} \text { und } \mathrm{H}^{2} \text {. }
\end{aligned}
$$

\section{Beitrag zur Kenntniss der Opiumbasen.}

$$
\text { Von } 0 \text {. Hesse. }{ }^{*} \text { ) }
$$

Von den vielen Verfahren, die zur Darstellung des Mor.phins empfohlen wurden, wird das von Robertson angegebene und von Gregory verbesserte Verfahren als dasjenige bezeichnet, nach welchem sich alle übrigen Opiumbasen gewinnen lassen sollen. Dieses Verfahren besteht bekanntlich darin, dass die Alkaloïdsalze des Opium durch

*) Alচ Separatabdruck aus den Annal. d. Chem. u. Pharm. VIII. Supplementband, 3. Heft, (Januar 1872) vom Herrn Verfasser erbalten. Mit Hinweglassung der Zahlenbelege f. d. Analysen hier wieder gegeben.

H. $\boldsymbol{L}$. 